\title{
Etude de l'habitat et scientificité de la géographie
}

S'approfondissant au fil des années, la géographie de l'habitat a fini par aboutir à une découverte capitale: l'inexistence de son objet tel qu'il avait été défini aux origines.

\section{Situation de la géographie de l'habitat}

\section{En France}

Ce résultat est le fruit de sa rigueur. Renonçant à renouveler les feux d'artifice d'intelligence ou de sensibilité d'un Vidal de la Blache ou d'un Jean Brunhes, la géographie de l'habitat avait cru trouver une base solide vers 1925 en adoptant la voie étroite du fonctionnalisme d'Albert Demangeon. La maison était un outil de travail lié à un certain type d'exploitation agricole, et l'habitat, défini par le groupement ou la dispersion des maisons, s'expliquait par la force ou la faiblesse des liens communautaires noués autour de l'exploitation du sol.

Bien qu'on ose rarement le reconnaître, cinquante ans de recherches ont ruiné cet édifice.

a) En ce qui concerne la MAISON tout d'abord: au fil des années, on a accordé une importance croissante aux aspects non fonctionnels: en 1948, PIERRE DEFFONTAINES ${ }^{1)}$ souligne l'importance des facteurs culturels et notamment religieux; en $1951 \mathrm{M}$. TRICART ${ }^{2)}$ croit sauvegarder l'apport de Demangeon en distinguant entre «Le plan et l'aménagement, adaptation aux conditions économiques et sociales», et "La construction, adaptation aux conditions physiques». En fait, définissant la maison par la rencontre de ces deux déterminismes indépendants l'un de l'autre, à quelques contraintes techniques près, il contribue à ruiner l'«idée féconde» de Demangeon, qui organisait tout autour du plan et de la fonction. Bientôt c'est le «fonctionnalisme» luimême qui sera attaqué de face: dans un article sur les «Transformations de l'habitat rural en Champsaun» M. LIVET ${ }^{3)}$ montre que l'«outil de travail», obéissant à sa logique propre, est finalement assez indépendant de ... la maison. Il rejoint en cela une constatation que l'on avait pu faire depuis longtemps: en milieu rural, l'amélioration de l'outil de travail est presque toujours l'objet essentiel de l'attention du paysan, la maison est prise telle qu'elle est, et transformée en dernier, sauf exceptions. En 1972, Pierre Deffontaines tirait avec courage les conséquences de ces recherches: il renonçait à faire une géographie de "la maison», qui n'était plus pour lui, qu'un assemblage de «dispositifs»: "La maison nous apparaitra ainsi surtout comme un assemblage d'agencements qui ont chacun leur domaine de répartition et leur évolution particulière; ils ont été regroupés en associations diverses pour donner des types d'habitation, mais ceux-ci, en fin de compte, ne sont que le résultat de multiples enchevêtrements et hybridations de dispositifs, et ce sont ces derniers qui, dans l'étude de la maison, nous paraissent fondamentaux»4). C'en était fait de la maison comme objet d'études géographiques.

b) l'HABITAT, comme étude du groupement et de la dispersion des maisons, n'est guère en meilleure posture. Déjà en 1950, M. TRICART portait un coup sévère en remarquant «l'opposition entre habitat groupé et dispersé est surtout un fait du peuplement de race blanche»s). Bien plus, en démontrant que le hameau est une forme de groupement, il ôtait à la dispersion une grande part de son étendue et de son intérêt. Réduite à peu de choses dans son domaine d'application, la problématique "groupement-dispersion» était enfin remise en cause dans son sens profond: M. DELVERT, dans son cours de Géographie tropicale 1973-1974 à l'Université de Paris-Sorbonne, montre que le lien groupement de l'habitat - force du lien social - agriculture organisée par la communauté - est tout à fait l'exception en Asie Tropicale, ne trouvant un correspondant parfait qu'au Viet-Nam. Ailleurs on peut trouver bien sûr ces éléments, mais ils ne se recouvrent pas dans l'espace: ainsi dans le cas de Bali, étudié par CLIFFORD GEERZ ${ }^{6}$ ), le "Bandjan", village groupé, est une unité sociale de vie intense et de puissante emprise juridique; mais la forte organisation de l'agriculture, liée à l'irrigation très sophistiquée, se fait en dehors de ce cadre: les "subak» regroupent les paysans de divers villages intéressés à une zone d'irrigation, en dehors du cadre de l'habitat. On pourrait multiplier les exemples.

Il n'est pas jusqu'à l'urbanisation du monde développé qui ne contribue à rendre désuète la problématique groupement-dispersion, en donnant à l'intégration urbaine par les mouvements pendulaires et l'in-

Jean-François Robert, Institut de géographie, Faculté des sciences, bd. Pérolle, 1700 Fribourg. 


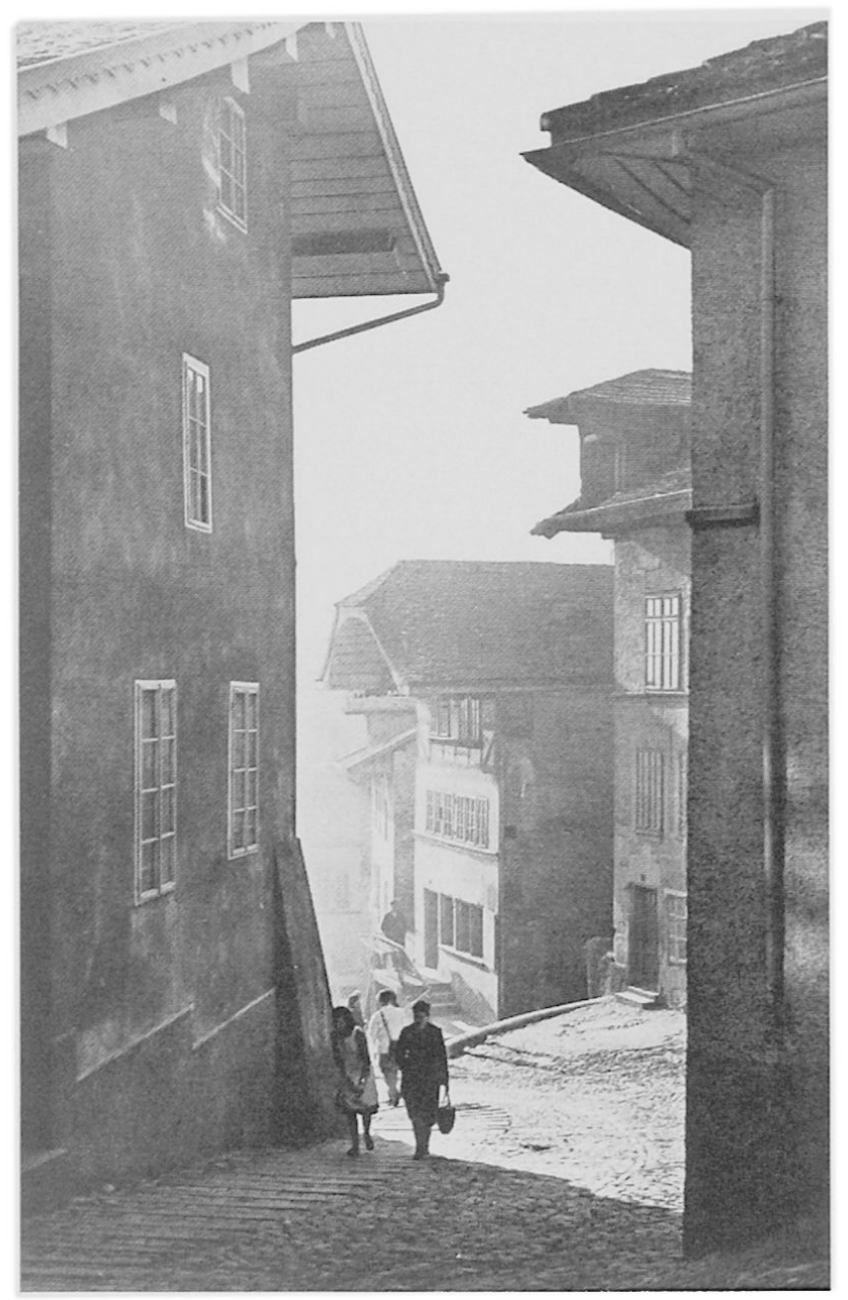

Fig. 1 «Le Court-chemin» à Fribourg

\section{Le double visage de la géographie de l'habitat:}

objectivité: une voie purement résidentielle, ignorée par la circulation générale; des maisons anciennes relativité: charmes et contraintes d'un site difficile; enracinement des habitants mais aussi peutêtre précarité de leur situation.

(Photo: Benedikt Rast, Fribourg)

troduction du confort la prépondérance sur le lien de l'habitat avec la vie rurale.

La géographie de l'habitat en France a donc connu récemment une crise profonde, mais nous pensons le prouver plus loin, vivifiante.

\section{Hors de France}

Ailleurs cette crise a été moins violente, parce qu'on avait accueilli avec plus de réserve peut-être le fonctionnalisme demangeonnien. Ainsi la notion d'habitat telle qu'elle ressort du livre de M. NIEMEIER «Siedlungsgeographie» ${ }^{7)}$, est-elle beaucoup plus large que celle des géographes français; avec la notion œkoumène, elle englobe, outre les études morphologiques, celle des genres de vies, et des fonctions économiques, réconciliant l'étude de l'habitat avec celle de la géographie urbaine, comme avaient tenté de la faire vers 1951 Jean Tricart et Max Sorre. Comme eux il s'approche ainsi d'une idée de l'habitat, synthèse à l'intérieur d'une géographie qui serait elle-même la science de synthèse par excellence. Mais, comme eux, il risque, en voulant trop embrasser, de perdre au niveau de l'efficacité pour la recherche ce qu'il gagne en étendue. Toujours est-il que les types d'habitat, très synthétiques, et par là même datés et localisés, qu'il définit, échappent aux reproches que l'on peut faire à leurs équivalents français, trop généraux du fait de l'emploi d'un nombre trop restreint de critères.

Dans le monde anglophone, un ouvrage remarquable d'AMOS RAPOPORT ${ }^{8)}$ parvenait récemment à des conclusions fort proches de celles de Pierre Deffontaines, malgré une approche radicalement différente: pour M. Rapoport, la maison est la réponse à un ensemble de questions, posées par la société et la nature sous forme de besoins culturels fondamentaux et de contraintes, modifiantes, liées au milieu naturel ou aux techniques de construction.

Déplaçant l'«idée féconde» de Demangeon de l'économie vers le social, A. Rapoport explique ainsi beaucoup de choses, et montre en outre que la maison fournit une partie seulement des réponses aux questions posées par la société, en concurrence avec d'autres institutions. Mais par là même le monde de A. Rapoport se révèle un monde plein, il ne connaît pas le vide: toutes les réponses sont adaptées à leur question, et toute question a trouvé sa réponse.

Le point commun de l'habitat-synthèse de Niemeier et de l'habitat-réponse de Rapoport, c'est qu'ils nous donnent des géographes heureux. De l'objet qu'ils étudient est exclue d'avance toute contradiction essentielle qui risquerait d'en mettre en cause la scientificité. Or, parce qu'elle est aussi une réalité humaine, la réalité géographique est minée par le ver de la contradiction.

\section{Exigences fondamentales et modalités d'une géographie de l'habitat}

«Le vieux testament est double» nous dit Pascal. La réalité géographique est à son image. Elle est ce qu'elle est, objectivement, telle que l'histoire et la géologie l'ont faite et l'expliquent tant bien que mal. Mais elle est aussi ce que nous ressentons, ce que nous vivons, ici, maintenant. Bien pis, elle est tout cela à la fois, ensemble et contradictoirement. Elle est l'unité de la contradiction.

\section{Exigences}

L'approche du géographe en est plus ou moins affectée. l'étude du paysage tolère dans un premier moment la seule approche objective et génétique. En géographie économique, cela est plus difficile: déjà Jean Brunhes nous suggérait d'utiliser l'idée bergsonienne de «direction de l'attention", qui rend aussi bien compte du sens profond de l'action économique, que des œillères qui la limitent au grand étonnement de l'observateur «objectif». 
Mais quand il s'agit de l'habitat, c'est-à-dire du cadre intime et familier de notre vie quotidienne, la prise en compte du vécu, et donc de l'ambiguité du réel, devient une ardente obligation. Ni le paysage, même construit par l'homme, ni l'homme, ni même les hommes, dans leurs rapports multiples qu'étudient les sociologues, ne suffisent à définir le champ d'études de l'habitat. C'est notre séjour habituel en tant que relation, être-là réciproque des hommes et des choses dans un cadre restreint, qui constitue l'habitat. L'habitat est présence, avec ce que cela comporte sur le plan spatial: la proximité, et sur le plan temporel: le temps présent. Comment l'étude de la présence peut-elle être scientifique?

La première condition est de regarder la difficulté en face, en refusant de l'éluder, comme nous le conseille JEAN FOURASTIÉ dans son livre sur «les conditions de l'esprit scientifique»9). La notion de présence est objective, elle est localisation; subjective, elle est le sens que prend cette localisation, c'est-à-dire le rôle qu'elle joue pour les habitants. Si l'on veut un mot unique qui résume ceci, celui de "place occupée par» convient le mieux: lui aussi a le double sens de localisation et de rôle.

Mais présence de quoi, place occupée par quoi? Présence de l'homme au monde, place occupée par le monde ou l'espace dans la vie de l'homme? Trop général: ce serait identifier la géographie de l'habitat à celle de l'espace vécu, dont elle n'est qu'un élément. Reconnaissant comme le veut Fourastié la fondamentale ambiguïté du réel, la géographie de l'habitat ne saurait préserver un minimum de scientificité qu'en privilégiant sur tous les autres points, les limites les plus strictes et les instruments les plus simples, le plus univoques.

Au niveau de son domaine, il nous semble, et nous l'avons suggéré, que le domaine adopté doit être celui de la vie quotidienne: avec la plus grande répétivité, c'est aussi le domaine de la plus grande intimité entre l'homme et le monde. La sphère des déplacements quotidiens sera le domaine de la géographie de l'habitat. Quel sera le point de départ? Etudierons-nous la place de l'homme dans l'espace quotidien? Non. Le mot «place» contient déjà toute l'ambiguïté liée à la présence de l'homme; n'aggravons pas la difficulté. Contentons nous d'un objet essentiel à l'homme: la maison, puisqu'il s'agit d'habitat? Non. Deffontaines en a montré, avec la richesse, l'incohérence. Il nous faut plus abstrait, plus univoque: le logement. La géographie de l'habitat étudie la place du logement, ou plutôt des logements, dans l'espace de la vie quotidienne, sur le mode objectif et sur le mode relativiste.

\section{Modalités}

a) Sur le mode objectif, ce qui ne posera guère de problème de scientificité: localisation des logements dans l'espace quotidien, c'est-à-dire d'abord dans le triangle théorique Logement-Travail-Echange, trois pôles dont le rapprochement donnera le modèle classique du village groupé, et dont l'écartèlement nous conduira vers l'habitat dispersé du bocage mais aussi, selon les cas, vers la vie «en miettes» des agglomérations urbaines à fort pendularisme. Puis on étudiera la localisation des logements les uns par rapport aux autres, ce qui suppose l'étude morphologique des types de maisons, individuelles ou collectives, voire disloquées quand elles ont une annexe temporaire (de travail: chalet d'alpage, ou de loisirs: résidence secondaire). Etude aussi, classique, des types de villages ou quartiers, selon leur forme ou leur ordre. Etude enfin de la localisation au regard du milieu environnant, naturel et construit, c'est-à-dire du site, facteur de confort et de personnalisation d'un ensemble habité, facteur aussi d'accessibilité plus ou moins aisée.

On voit par là que l'étude «objective» de la localisation est déjà placée, comme on pouvait le deviner en considérant le point de départ choisi (logement-travailéchange) sous le signe du dialogue entre l'absolu et le relatif, l'objectif et le vécu. Il en ira de même pour l'étude plus «relativiste» du rôle de l'habitat.

b) Relativiste dans son essence, l'étude du rôle de l'habitat peut se subdiviser à son tour entre un aspect plus «objectif»: la fonction, et un aspect plus «relatif»: le sens. Pourquoi cette distinction? Parce que nous avons un corps, nous percevons nécessairement les choses sous le signe de la présence ou de l'absence; le rôle d'une chose présente sera généralement différent du rôle d'une chose absente. Prenons un exemple: tel appartement loge une personne, c'est sa fonction; mais il s'agit d'un travailleur immigré dont la famille est à mille kilomètres de là; c'est l'absence de cette famille qui va donner son sens au logement, qui sera un simple abri pour la nuit dans un espace voué au travail, et non pas un véritable foyer.

Certes, heureusement, la fonction et le sens peuvent converger vers le bonheur des individus, mais le caractère fondamental en géographie de ces notions de présence et d'absence nous imposent cette distinction. L'étude de la fonction sera donc l'étude de tout ce que nous apporte en fait l'habitat; sur le plan matériel, ce sera l'étude du confort de la maison (surface disponible par personne, équipement, agencement des pièces) mais aussi de l'équipement du village ou du quartier (services, loisirs); sur le plan humain, ce seront les fréquentations permises par l'habitat: la famille d'abord (sa taille, sa composition) mais aussi, selon les cas, les connaissances et les amis; ici ce sera le sociologue qui renseignera le géographe. Il lui indiquera aussi le mode d'organisation (sociétés, etc.) de cette sociabilité et sa portée spatiale; enfin, ce que l'habitat offre sur le plan matériel ou humain doit être affecté d'un coefficient essentiel: celui du temps passé. La fonction de l'habitat n'est pas la même pour l'enfant qui jouera, selon l'expression de Bachelard, «de la cave au grenien», pour la femme au foyer, dont la maison est l'horizon habituel, et pour le travailleur absent pendant le jour et qui préférera le bistrot, la télévision ou ... son lit. 
On a appris récemment que le Suisse consacrait en moyenne quatre heures par jours aux «mass-media». Cela veut dire aussi que pendant ce temps, le cadre restreint de son habitation se dilate en fait aux dimensions du canton ou du pays, sinon du monde. Au niveau de la fonction, le temps est donc déjà une dimension essentielle de l'espace. Il l'est plus encore au niveau du sens.

L'étude du sens de l'habitat est infiniment délicate. Il faut se garder de la pousser jusqu'à l'affirmation que telle forme d'habitat a tel sens, et surtout de l'exprimer en termes de bonheur ou de malheur, car les replis de notre âme sont imprévisibles. Sur la base des études des psychologues et des sociologues, le géographe a toutefois la possibilité de détecter ce qui aura statistiquement de l'importance, et donc ce qui au niveau individuel aura une forte chance d'être important.

Ainsi J. BASTIÉ, dans son ouvrage sur «La croissance de la banlieue parisienne»10), remarque-t-il, sur la base d'enquêtes, que les grands ensembles sont appréciés de façon radicalement différente selon que l'habitant est un ancien "mal-logé» de la périphérie ou au contraire un ancien «Parisien» obligé de quitter la ville; à partir de ce moment une étude scientifique devient possible, qui le restera tant qu'elle ne prétendra pas descendre jusqu'au niveau du bonheur individuel.

L'exemple pris chez Bastié est aussi une bonne illustration du lien que nous établissons entre sens et $a b$ sence: ici c'est le passé des habitants qui pèse de tout son poids sur le présent. Plus généralement, on peut voir plusieurs formes de l'absence:

Par rapport au temps, ce sera le passé ou le futur. Passé des individus, comme on l'a vu ci-dessus, futur aussi, comme par exemple ce que représente une ville universitaire pour des étudiants (bien que certains d'entre eux vivent surtout dans le présent...). Pour le groupe social tout entier, ce seront au fond les notions d'enracinement (avec tous ses symboles: vieux pont de bois ...) et de frontière (au sens de l'Ouest américain, mais applicable à tous les lieux, par exemple urbains, où l'avenir se crée sous nos yeux).

Par rapport à l'espace, l'absence peut être celle d'une partie de la famille; mais ce peut être aussi, en général, l'absence de ce qui est valorisé: dans les banlieues étudiées par Bastié, «La gare et la rue qui y conduit sont les centres de la vie, les lieux de recontre, la porte constamment ouverte sur le monde attirant de la grande ville et de ses activités, à la fois si proche et si différent».11) Inversement l'«ailleurs» peut être le faire-valoir l'«ici», l'étranger inconnu et menaçant qui fait apprécier le «chez-soi».

On voit qu'à ce niveau il ne peut guère y avoir de scientificité des résultats, qui ne seront jamais totalement adéquats au réel, mais il peut y avoir scientificité de la méthode, par la recherche de ce qui est important, par la conscience critique des limites de nos instruments d'enquête.

Nous approchons ainsi d'une autre dimension de la scientificité, celle que rappelait J. L. PIVETEAU dans son ouvrage sur «La structure urbaine du canton de Fribourg»12): La conscience que l'on doit avoir des limites d'une entreprise. La géographie de l'habitat, pour être scientifique, doit reconnaître ses limites.

\section{Scientificité et limites de la géographie de l'habitat}

Au fil des pages qui précèdent, on n'aura pas manqué de relever, peut-être avec surprise, deux constantes: tout d'abord, dans l'étude de l'habitat, le seul fil conducteur, la seule liaison systématiquement étudiée, c'est la complicité nouée, à partir de l'habitat, entre l'homme et un certain espace restreint qui lui est familier. Et la deuxième constante, c'est que nous demeurons sans cesse au niveau de la description, sans aborder l'explication.

Ce n'est là le fruit du hasard ni d'une quelconque négligence.

\section{La géographie de l'habitat étudie des régions et non des lois}

Si la loi est l'«expression du rapport nécessaire qui lie entre eux des phénomènes» (Encyclopédie Larousse), on voit que la géographie de l'habitat telle que nous la concevons n'étudie pas des lois, et ceci pour deux raisons: d'une part, ce ne sont pas les rapports des phénomènes entre eux qui nous intéressent, mais leurs rapports avec nous, c'est-à-dire avec un groupe d'habitants liés par un espace vécu commun. La localisation de l'habitat n'a de sens que par rapport aux habitants. D'autre part, il est dans un premier temps indifférent au géographe que ce rapport soit nécessaire ou non, pourvu qu'il existe. Le géographe accorde une valeur égale aux fruits du hasard et à ceux de la nécessité.

$\mathrm{Si}$ le géographe ne cherche pas à découvrir des lois, que cherche-t-il? Il cherche à découvrir un rapport, certes, plus précisément un ordre, simplement cet ordre ne relie pas entre eux des phénomènes par un lien nécessaire, mais relie, d'un lien aléatoire ou nécessaire, des phénomènes à un groupe d'habitants. Cet ordre qui s'organise dans l'espace autour d'un groupe d'habitants, c'est ce que l'on appelle une région. Le produit de notre activité scientifique est bien la découverte d'un rapport, mais ce rapport est une région et non une loi.

Est-ce à dire que le géographe serait condamné à la contemplation de l'unique, du local, bref de l'idiographique? Non. De la répétion de formes essentielles du rapport homme-espace, le géographe peut tirer des typologies; à une condition, c'est bien le rapport vécu homme-espace qui doit être ressemblant, et non tel aspect de l'homme seul ou de l'espace seul, et de plus il faut une convergence de critères, qui ne peut guère se trouver dans des régions trop éloignées; aussi considérera-t-on comme déjà très tolérante la remarque de PIERRE GEORGE: «Les généralisations d'un continent à l'autre sont dangereuses» ${ }^{13)}$. 
Au fond, on ne peut établir de typologie qu'à l'intérieur d'un espace vécu commun, même s'il est un peu plus lâche que celui des unités élémentaires d'habitat, bref, dans le cadre d'une région d'échelle supérieure. La généralisation en matière de géographie de l'habitat ne consiste pas à établir des lois, mais à reconnaître l'emboitement des régions élémentaires dans des régions plus vastes.

Il ne s'agit donc pas d'un cantonnement dans l'idiographique seul, mais d'un dialogue du régional et du général, dans lequel, comme l'avait vu M. LE LANNOU dès 194914), le régional est toujours la fin et le général le moyen.

Cela veut dire que le progrès scientifique, en matière de géographie, consiste dans l'approfondissement de la notion de région: cela veut dire aussi que la vérification, a priori difficile quant au contenu des rapports hommeespace puisque l'on aura mis en œuvre pour la découverte tous les moyens disponibles, pourra se faire par une confrontation systématique des limites spatiales, c'est-à-dire des limites régionales.

Tout cela va nous permettre désormais de répondre plus facilement à la second question: pourquoi privilégier la description par rapport à l'explication?

\section{Seule la description géographique pose des problèmes spécifiques de scientificité}

Quand le géographe cherche à expliquer, que fait-il? Il ne se penche plus sur le rapport présent, original, "homme-espace», mais sur le rapport entre un état de choses donné (les rapports présents homme-espace) et tous les autres phénomènes susceptibles de contribuer à l'expliquer. Il établit le rapport de plusieurs phénomènes entre eux: son problème est désormais le même que celui de ses collègues, historiens sinon naturalistes. La scientificité des explications est un problème commun aux sciences de l'homme, il n'y a rien de particulier à la géographie. Il y faut tenir compte de tout, y compris des effets de distance et de configuration spatiale par exemple, mais l'historien et même le physicien doivent aussi en tenir compte, sinon leur explication sera fausse. Dès lors, il ne faut pas hésiter à en tirer la conséquence: l'explication des phénomènes géographiques n'appartient pas en propre aux géographes. Elle peut être menée par une personne étrangère à notre problématique, sur la base de ce que nous lui donnons à expliquer.

Cela ne veut pas dire que le géographe ne doit pas chercher à expliquer, mais simplement que ce n'est pas sa façon d'expliquer qui fait son originalité sur le plan scientifique.

La géographie de l'habitat ne découvre pas des lois mais des régions, elle a un privilège au niveau de la description mais non de l'explication: sa scientificité est au prix de la reconnaissance de ces limites. Ces limites sont-elles aussi celles de toute la géographie?

\section{Conclusion: Les limites de la géographie}

Au delà de la géographie de l'habitat, on voit que la géographie économique, avec sa double obédience au domaine des lois qui régissent les choses et des régions qui sont l'expression de l'action humaine, participe encore de notre vision «asymétrique» des rapports entre l'homme et le monde. Simplement, la définition des régions économiques sera plus délicate encore que celle des régions d'habitat, qui devra la précéder.

Il n'y a plus de géographie dès lors que l'idée d'un «tout terrestre» homogène l'emporte; ainsi l'écologie, cherchant à établir un circuit dont l'homme n'est plus qu'un maillon, n'est-elle plus géographique à notre sens: un réseau d'équations y suffit. Au contraire, une géographie physique orientée vers la notion de «paysage», qui déjà suppose le regard de l'homme, appartiendrait à notre discipline.

$\mathrm{Au}$ fond, si le monde n'était que le fruit d'un jeu du hasard et de la nécessité, la géographie serait inutile: une écologie historique suffirait. S'il nous faut une géographie, c'est que le monde est plutôt la scène, pour reprendre l'expression de Mativaux, d'un vaste «jeu de l'amour et du hasard». Ce qu'exprimait d'une autre façon le philosophe de la science Gaston Bachelard, en disant que «le monde est intense avant d'être complexe».

\section{Références}

1) DEFFONTAINES, PIERRE: "Géographie et religions», Gallimard 1948.

2) TRICART, JEAN: Cours de géographie humaine, tome I: Habitat rural, 1950-1951 policop.

3) LIVET, R.: «Transformations de l'habitat rural en Champsaun in NOIROIS no. 63, 1969.

4) DEFFONTAINES, PIERRE: «L'homme et sa maison», Gallimard 1972.

5) TRICART,JEAN: op. cit. p. 92.

6) GEERZ, CLIFFORD: in "Villages in Indonesia», sous la direction du Dr Koentjaranningrat.

7) NIEMEIER: «Siedlungsgeographie», Westermann 1967.

8) RAPOPORT, A.: «Pour une anthropologie de la maison», Dunod 1972.

9) FOURASTIÉ, J.: «Les conditions de l'esprit scientifique», coll. Idées, Gallimard 1966.

10) BASTIÉ, J.: «La croissance de la banlieue parisienne», PUF 1964.

11) BASTIÉ, J.: op. cit. p. 24.

12) PIVETEAU, J. L.: «La Structure urbaine du canton de Fribourg», Fribourg, 1970, p. 2.

13) GEORGE, P.: «Précis de géographie rurale», PUF 1963 , p. 183.

14) LE LANNOU, M.: «La géographie humaine», Flammarion 1949. 